\title{
Decrease of Power Consumption Utilizing IOT
}

\author{
Shiela David, Akshara P, Shagun Dixit, Sai Purnimaa
}

\begin{abstract}
As electricity consumption is very high so using IOT, reduction of electricity consumption is tried company nowadays but the use of it in extreme levels results as a damage to environment and personal financial conditions.Involving fields of application and Internet of things sensors are made. In this paper an architecture is introduced, that helps the smart cities to save resources for future. Consumption of electricity in a way is destroying the environment so this paper helps in saving the cost of electricity as well as saving the environment using wireless connections. Finally a new system is proposed keeping in mind the old one and its challenges. This system overcomes all the challenges and difficulties faced by the old one. To implement the framework electrical appliances such as lights, fans, ac etc are incorporated within the proposed system of reduction of electricity consumption.
\end{abstract}

Index terms- Internet of things; IOT; Reduced electricity consumption; Saving the cost and resources; Smart cities; Wireless connection

\section{INTRODUCTION}

In India, the electricity consumption has drastically changed in past 5 years. In 2015-16 the electricity consumption was 6.76 GW whereas in the next year 2016-17 it became 12.28 GW which brings to the conclusion that its being rapidly increasing every year. Being the second

most populated country in the world, India is using electricity at its peak and for sustainableusage there is a need to save resources like electricity.

The main motive of this paper is to save electricity using IOT. Internet of things network coordinates with four unmistakable parts: sensors, availability, information preparing, and a UI . To begin with, sensors collects information from their specific condition. This can be a basic as measuring temperature or can be the recording of videos. The sensors can be connected to the cloud via different ways including: cellular, satellite, Wi-Fi, Bluetooth, or connecting directly to the internet via Ethernet.When the data is sent to the cloud,,the role of progamming is done. One of the simple example can be, checking the temperature in a particular range Or on the other hand it can be like utilizing the PC vision to record the different objects and movements .Next, the stored information is made secure to the end-client. This can be made possible by alreting the particular client by setting an alarm (email, content, warning, and so forth). For instance, a notification will pop up if the temperature is disturbingly high in the organization's cold storage.Additionally, a client will have an interface that helps them to monitor the framework for $24 * 7$. For example, a client can check the video footage in their home by means of an application or a browser.
The existing system of measuring the usage is traditional and complex. This involves an electricity meter which reads the total consumption where a manual calculation is done for paying the bill altogether. This paper overcomes the challenges faced by the existing system by introducing a mobile application and sensors connected through cloud by IOT which are monitored by the consumer 24/7 anywhere-anytime.

\section{GOAL OF THE PAPER}

This paper overcomes the problem faced by the existing system and helps in reducing the consumption of electricity. The project deals with sensors connected with each appliances by iot sharing all the information to the cloud. Sensors are intended to meet the carbon reduction and conserving energy. The circuit involves arduino energy monitoring system measuring the current using ct sensors.

\section{RELATED WORKS}

\section{[3.1]Smart security system}

The framework comprises of a camera, voice sensor/mouthpiece ,movement/action sensor and a LTE/Wi-Fi module which is interfaced with the core of the framework, processor. The fundamentalsof this framework is the capacity to remotely deal with one's property anytime. With this IOT based keen storage, one can screen, get alarms, advise if there should be an occurrence of crisis from anyplace on the planet utilizing portable application by means of cloud network day in and day out.

The plan is a straight forward,dependable and powerful security and robbery control.This gives low control reconnaissance arrangement, which will be successful for people to screen their storage spaces by means of moment video alarms upon any movement identification inside the storage and the area can be observed consistently

Well being is an incredible risk if any programmer can get to the cloud, all the data can be transmitted and can likewise be effectively stole from the proprietor.Security is the primary worry as the information being transmitted, the danger of protection increments since the recordings are stored.

[3.2]Building an IOT Infrastructure.

It is making an IOT programming which is simply open hotspot for ensuring that its not claimed by a solitary association rather a network and we can create and send and gives intergration.For Security

purposes, acurators are utilized ie, they distinguish and tells about the un anticipated conduct. It gives different levels of access to sensor information, from the most secure to the primary sensor has actualized a powerful visibility restricted layer to enable versatile security arrangements to be conveyed just as consultations to manage with the amount of standards that must be resolved.

It provides apparatuses for the end client to make a virtual sensor. To deal with the huge measure of data,from the inserted sensors information 


\section{Decrease of Power Consumption Utilizing IOT}

examination is utilized by diminishing the power use as principle are sent to the sensors.Directions are transmitted to sensors to gather information in a specific proportion are arranged continuously utilizing force utilization examination. The design must fulfill every single current solicitation for information. Thus, the GIoTTO is solid client support for the establishment, upkeep, and control of an IoT situation.

The challenges in ensuring the security of IoT devices are furthermore because of remarkable heterogeneity, absence of organization, and ineptitude of customary assurances like end-have firewalls and antivirus programming. IOT protection difficulties come from sensors legitimately or in a roundabout way releasing private data about clients, frequently obscure to them.

Security in IoT means giving access control systems and strategies and having the option to authorize them, especially notwithstanding the huge number of heterogeneous gadgets.

[3.3] Smart homes using IOT

Arranging a negligible effort,extensible, adaptable remote splendid home automation structure using Iot which uses the joining of remote correspondence, cloud frameworks organization to offer customers to control an arrangement of devices from remote zones by giving a simple to utilize interface and straightforwardness of foundation.

Control home application by procedures for android application : to develop an android application that features decision of manual or altered system for controlling home machines and give status of the home condition. Sensor based control of contraptions: to make a framework that controls home

It is costly and can't be moderate for all class individuals .There might be some expectation to learn and adapt for non-tech individuals. Keen homes without web isn't solid and we cannot screen without web.

\section{[3.4] IOT systems for cities}

It bargains about proposing sensquare an engineering which is about committed administrations, by incorporating heterogeneous information sources together, and making them accessible to the end client with an available interface. The clients have then various conceivable outcomes to make their own administrations, through a web interface or through a portable application.

Community oriented CBM presents an increasingly mind boggling structure of client pool - for example, it can incorporate natives, partners, makers and purchasers - and can be additionally classified. This is the situation of Community based monitoring(CBM), which are incredible when dealing with every one of the elements actualized inside them, anyway they act as IOT islands which have next to zero interoperability with others.

This can prompt information excess and inaccessibility when managing ecological observing, for example at the point when information is intriguing for the normal benefit.

Heterogeneous information gathered from either clients or open information sources regularly has the downside of being unlabeled and scanty and, in this manner, its significance is not really understandable.

Crowd sensing efforts need to manage the resident interest, typically cultivated through client inspiration, low quality information and area mindfulness information mining, which are both testing and right now contemplated issues.

[3.5]Cell phone processing utilizing IOT

Wireless fused sensors offer pushed organization which when gotten together with web-enabled true contraptions arranged near the versatile customer having the ability of updating the general customer learning acknowledgment and experience engaging progressively taught choices and better decisions.

Cell phone incorporated sensors offer propelled capacities, for example, estimating vicinity, speeding up and area or record sound/commotion, sense electromagnetism or catch pictures and recordings.

Demonstrates the significance of participatory detecting for our day by day lives, to be specific its effect on climatic change.,GPS-prepared cell phones are utilized to photo diesel trucks, so as to comprehend network presentation to air contamination.

As to mechanization and control, it is estimated that Internet innovation, in accordance with IOT, will turn into the future standard, offering propelled inter operability between heterogeneous home gadgets and apparatuses.

Dependability ends up significant particularly in well being checking applications that include body territory systems. The comparing portable applications should most likely distinguish inconsistencies and deficiencies at the information, which could make false cautions. Security is a top need in portable processing, and turns out to be increasingly significant when associating with the IOT gadgets.

[3.6] Architecture of internet of things

The Architecture using this will help an enormous measure of advancement, proficiency, and quality. Interfacing creation, restorative, car, or transportation frameworks with IT frameworks and business-basic data will give huge incentive to associations.

Network and correspondences are significant. This may include coordinated network (unicast) or information accumulation and data scattering to numerous accomplices (multicast and anycast). Device the executives must give arrangements once a gadget is included or a gadget setup changes and should be spread to different gadgets. Data gathering, examination, and activation are significant for separating data and learning for offering administrations. Scalability is essential to deal with expanded preparing volumes for various establishment sizes. Security highlights are important to give trust and protection and are required for all parts of the IOT.

The IoT is wealthy in methodologies, ideas, and structures. Different activities have just conveyed IoT models, structures, and apparatuses. In any case, a further union of methodologies and modern gauges is required for improvement. This raises issues about the key IoT empowering agents. One key empowering agent is proficient, secure correspondence. Numerous innovations exist to actualize the correspondence stack. Low-control remote systems that require little usage exertion are essential, for which security is a significant factor.

[3.7]Standardizing IOT

Sensors around us are increasingly becoming more pervasive and attempt to fulfill end clients' needs, thus providing ease of ease of use in our regular exercises.

This interconnection provide saw whole range of information (natural setting, gadget status, vitality utilization, and so on.) that can be collected,aggregated,and then shared in efficient, secure, and protection mindful way. As these gadgets are associated with the Internet, they can be come to, and oversaw at whenever and at

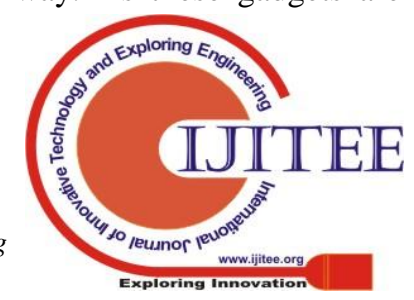


any place. The current scene of IoT is filled with a different scope of remote correspondence innovations, for example, IEEE 802.15.4, WiFi, Bluetooth Low Energy (BTLE), and different other cell correspondence advances.

It propels the significance of security standards. The achievement of the World Wide Web benefited from a strong establishment based on an institutionalized convention stack comprising of the IP.This is significant that institutionalization guarantees interoperability The reasons that another convention is defined for compelled IP systems, rather than just reusing HTTP, is to incredibly diminish overhead in usage multifaceted nature and to lessen the data transfer capacity necessities. Such information decrease additionally expands unwavering quality (by diminishing connection layer fracture) and lessen inertness in run of the mill low-control lossy remote systems.

Deferrals in system access and correspondence are terrible since they lead to higher asset utilization. At long last, another significant measure is the quantity of messages traded within the sight of parcel loss. The DTLS convention usage was additionally inspected and assessed by tuning the bundle misfortune proportion as some UDP parcels will undoubtedly get lost because of system blockage and constrained system data transmission.

[3.8] IOT with interoperability

The touching off such an IoT environment, interoperability crosswise over stages must be empowered. Such interoperability will give engineers a chance to make applications by consolidating information from various platforms.Also, stages from numerous areas would then be able to be joined - for instance, a wearables stage with a shrewd home stage. An application will take a shot at top of various stages. Environment Architecture Model is a model of the essential biological system engineering. The distinctive IoT stages offer access to different sorts of things. Other than giving their own interfaces, the stages are improved with the BIG IoT API, a typical interface that offers the required functionalities for interoperability with different stages. The stages can work on the cloud level, mist level or gadget level.

The most existing and developing IoT stages offer heterogeneous approaches to get to things and their information. This causes interoperability issues when engineers plan to make all-encompassing, cross-stage, and cross-space applications, and it inevitably avoids the rise of energetic IoT biological systems. It additionally prompts obstructions for business openings, particularly for little creative undertakings, which can't bear to give their answer over different stages.

Implementation

A Network association is made between the remote PMC controllers and the IoT star programming by means of an Ethernet/4G Network, IoT star would then be able to speak with the remote controllers so it can be used to design the framework settings, actualize status checking usefulness, and perform firmware refreshes, which work regardless of whether the controller is a piece of a Private IP Domain arrangement condition, for instance, situated behind a Firewall or where you are utilizing a Dynamic Virtual IP.

The IoTstar application can be introduced on a standard PC stage and works as a Private Cloud the board framework for WISE/PMC/PMD controllers. It can likewise be introduced on a virtual machine(VM) stage dependent on Microsoft Azure, IBM Bluemix or Amazon AWS, and so forth., and capacities as a Public Cloud WISE/PMC/PMD controller the executives framework.

Then giving remote upkeep and firmware update benefits on WISE/PMC/PMD controllers, IoTstar can likewise be utilized to gather information from I/O channels just as recovering force information from $\mathrm{I} / \mathrm{O}$ modules, sensors, and power meters that are associated with the WISE/PMC/PMD controller, at that point transmit this information to a database. IoTstar permits status questions and shows computerization control and checking in a cloud situation.

By utilizing SQL database directions, IoTstar can be joined with a wide scope of cloud stage administrations and information investigation devices, for example, Power BI or Google Data Studio, and so forth., or with a SCADA framework to help rapidly make "IoT + Big Data" Cloud applications, just as altogether diminishing the time and cost.

\section{IMPLEMENTATION}

This guide subtleties how to fabricate a straightforward vitality screen on a breadboard that can be utilized to gauge how much electrical vitality used in home. It gauges current, however utilizes an accepted fixed an incentive for voltage $(220 \mathrm{~V})$ and ascertains evident power. Despite the fact that not as exact as a screen that estimates voltage just as current, it very well may be a technique usually utilized in economically accessible entire house vitality screens for reasons of effortlessness and cost.

$1 \times$ Arduino

Current detecting hardware

1 x CT sensor YHDC SCT-013-000

$1 \mathrm{x}$ Burden resistor $18 \mathrm{Ohms}$ if supply voltage is $3.3 \mathrm{~V}$, or 33 Ohms if supply voltage is $5 \mathrm{~V}$

$2 \times 10 \mathrm{k}$ Ohm resistors (or any equivalent worth resistor pair up to $470 \mathrm{k} \mathrm{Ohm)}$

$1 \times 10 \mathrm{uF}$ capacitor

A breadboard and some single center wire

The screen comprises of the present sensor (which delivers a sign relative to the mains current) and the sensor hardware that convert the sign into a structure the Arduino can use.

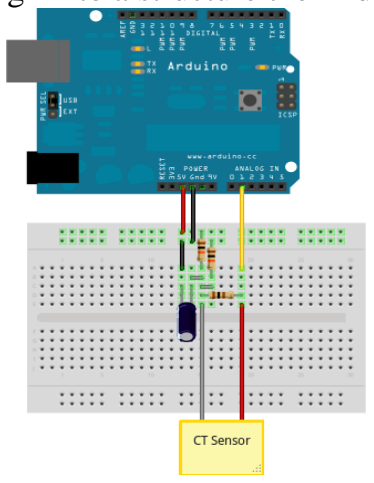

Assemble the parts per the outline above.

The sketch is the product that keeps running on the Arduino. The Arduino changes over the crude information from its simple contribution to comprehensible qualities, at that point sends them to the sequential port monitor.Download EmonLib from github and spot it in Arduino libraries organizer.

set two sections of qualities. Clear control on the left, RMS current on the right. 


\section{Decrease of Power Consumption Utilizing IOT}

\section{CONCLUSION}

Hence, This project helps us in reducing the electricity consumption in an inexpensive way and being eco friendly it improves the living standard with the help of IOT which is the new upcoming platform for all kind of technological advancement for a progressive world.

\section{AUTHORS PROFILE}

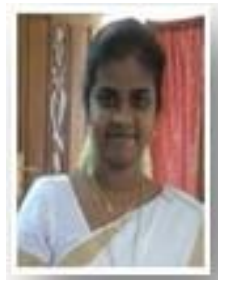

Mrs. Shiela David, M.E Assistant proffessor (O.G) Computer Science \& Engineering SRM Institute of Science and Technology Ramapuram Campus, Chennai

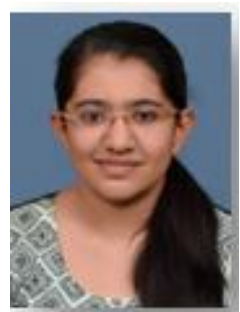

Akshara P, Btech CSE, III Year SRM Institute of Science \& Technology Ramapuram Campus, Chennai

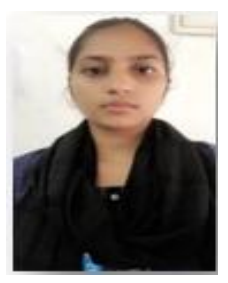

Shagun Dixit, Btech CSE, III Year SRM Institute of Science \& Technology Ramapuram Campus, Chennai 